\title{
BLOCKS OF RATIONAL REPRESENTATIONS OF A SEMISIMPLE ALGEGRAIC GROUP
}

\author{
BY STEPHEN DON KIN ${ }^{1}$
}

Let $G$ be a semisimple algebraic group over an algebraically closed field $k$. Any rational representation of $G$ gives rise naturally to a representation of the Lie algebra $L$ of $G$. If the characteristic of $k$ is zero then, by a classical theorem of Weyl, every finite-dimensional representation of $L$ is completely reducible. From this, it follows that every rational representation of $G$ is completely reducible. However, when the characteristic of $k$, say $p$, is not zero, there are always rational representations which are not completely reducible. The extent of the lack of complete reducibility is measured, in some sense, by the block theory of $G$.

We say that simple rational $G$ modules $M_{1}$ and $M_{2}$ are adjacent if both $M_{1}$ and $M_{2}$ occur as composition factors of some rational indecomposable $G$ module. A block is then an equivalence class of simple $G$ modules under the equivalence relation generated by adjacency. We shall also, less precisely, use the expression " $V$ belongs to the block $B$ " to indicate that each composition factor of the rational $G$ module $V$ belongs to $B$. Suppose that $\left\{B_{i}: i \in I\right\}$ is the set of blocks and that $V$ is an arbitrary rational $G$ module. Then $V$ has a unique $G$ module decomposition

$$
V=\sum_{i \in I} \oplus V_{i}
$$

such that $V_{i}$ is in the block $B_{i}$ for each $i \in I$.

Let $T$ be a maximal torus of $G, W$ the corresponding Weyl group and ( , ) a positive definite, $W$-invariant, inner product on $X(T) \otimes_{\mathrm{Z}} \mathrm{R}$, where $X(T)$ is the character group of $T$. Assume now that $G$ is simply connected and that the root system of $G$ is indecomposable (a description of the blocks in this case yields easily a description in the general case). The simple rational $G$ modules are indexed by the elements of $X^{+}$, the set of weights in $X(T)$ which are dominant relative to some fixed choice of system of positive roots of $G$. For an element $\lambda$ of $X^{+}$we denote by $L(\lambda)$ the simple rational $G$ module of highest weight $\lambda$. Each simple rational $G$ module is isomorphic to precisely one member of $\left\{L(\lambda): \lambda \in X^{+}\right\}$. Thus a block of rational representations of $G$ may be identified with a subset of $X^{+}$; for $\lambda$ in $X^{+}$we denote by $B(\lambda)$ the set of dominant weights $\tau$ such that $L(\tau)$ is in the block containing $L(\lambda)$.

Received by the editors February 12, 1980 and, in revised form, April 7, 1980.

AMS (MOS) subject classifications (1970). Primary 20G05; Secondary 20G10.

${ }^{1}$ Research supported by the Science Research Council of Great Britain. 
We denote by $\rho$ half the sum of the positive roots. The Weyl group $W$ has a natural action on $X(T)$, which we modify to obtain the 'dot' action, given by $w \cdot \lambda=w(\lambda+\rho)-\rho$ for $w$ in $W$ and $\lambda$ in $X(T)$. The 'dot' actions also plays an important role in the similar problem of sorting out the indecomposable modules in the category $\mathrm{O}$ of Bernstein, Gelfand and Gelfand. The analogy between that problem and ours was first pressed by Verma in [7].

For a dominant weight $\lambda$ we define $r(\lambda)$ to be the nonnegative integer such that $\lambda+\rho$ belongs to $p^{r(\lambda)} X(T)$ but not to $p^{r(\lambda)+1} X(T)$. We denote by $R$ the root system of $G$ and by $\mathrm{ZR}$ the subgroup of $X(T)$ generated by $R$.

The result we wish to announce is the following

THEOREM [4]. For any dominant weight $\lambda$,

$$
B(\lambda)=\left(W \cdot \lambda+p^{r(\lambda)+1} Z R\right) \cap X^{+} .
$$

An equivalent formulation of this is the statement that dominant weights $\lambda$ and $\tau$ are in the same block if and only if $r(\lambda)=r(\tau)$ and $\lambda$ and $\tau$ are conjugate under the 'dot' action of the affine Weyl group with respect to $p^{r(\lambda)+1}$.

The result was proved for the two-dimensional special linear group by Winter in [8] and for $G$ of arbitrary type and $\lambda$ " $p$-regular" by Humphreys and Jantzen in [5]. Results on the blocks of $G$ are also to be found in [2].

The easier half of our proof consists of showing that the set $(W \cdot \lambda+$ $\left.p^{r(\lambda)+1} \mathrm{ZR}\right) \cap X^{+}$is a union of blocks. Here we build on the recent work of Andersen [1] which implies the desired conclusion when $r(\lambda)$ is zero. We argue by induction on $r(\lambda)$ via results from the author's thesis [3]-though one could also use here the results of $\$ 2.4$ of [5]. In the second half of the proof we show that the elements of $\left(W \cdot \lambda+p^{r(\lambda)+1} \mathrm{Z} R\right) \cap X^{+}$belong to the same block. Of crucial importance here is the following observation: suppose that $\lambda$ is a dominant weight, $\alpha$ a simple root and suppose that $2(\lambda+\rho, \alpha) /(\alpha, \alpha)$ is equal to $b p^{r}+$ $a p^{r+1}$ for integers $a, b, r$ with $0<b<p$; if $\lambda-b p^{r} \alpha$ is dominant then it is in the same block as $\lambda$. Using this simple result one can go a long way (using the arguments of Jantzen in $\$ 5.5$ of [6] ) but for weights close to the walls of the dominant region something more is needed. We must rule out the possibility that there is some block $B$ such that each element of $B$ is close to some wall of the dominant region. This possibility is excluded by considering composition factors of indecomposable summands of $G$ modules of the form $L(\lambda) \otimes$ $L\left(\left(p^{n}-1\right) \rho\right)$ for $\lambda \in X^{+}$.

I am grateful to Professor R. W. Carter for his part in many illuminating discussions on this subject.

\section{REFERENCES}

1. H. H. Andersen, The strong linkage principle, J. Reine Angew. Math. 315 (1980), 53-59. 
2. E. Cline, B. Parshall and L. Scott, Cohomology, hyperalgebras and representations, J. Algebra 63 (1980), 98-123.

3. S. Donkin, Problems in the representation theory of algebraic groups, Ph. D. thesis, University of Warwick, 1977.

4. - The blocks of a semisimple algebraic group, J. Algebra (to appear).

5. J. E. Humphreys and J. C. Jantzen, Blocks and indecomposable modules for semisimple algebraic groups, J. Algebra 54 (1978), 494-503.

6. J. C. Jantzen, Über Darstellungen K̈̈nerer Frobenius-Kerne halbeinfacher algebraischer Gruppen, Math. Z. 164 (1979), 271-292.

7. D. N. Verma, The rôle of affine Weyl groups in the representation theory of algebraic Chevalley groups and their Lie algebras, Lie Groups and their Representations (I. M. Gel'fand, ed.), London, 1975.

8. P. W. Winter, On the modular representation theory of the two dimensional special linear group over an algebraically closed field, J. London Math. Soc. 16 (1977), 237-252.

MATHEMATICS INSTITUTE, UNIVERSITY OF WARWICK, COVENTRY CV4 7AL, ENGLAND 